Proceedings of SALT 24: 177-196, 2014

\title{
A superlative argument for a minimal theory of definiteness ${ }^{*}$
}

\author{
Elizabeth Coppock \\ SCAS, Uppsala University and \\ University of Gothenburg
}

\author{
David Beaver \\ University of Texas at Austin
}

\begin{abstract}
This paper argues that the distinction between absolute and relative readings of superlatives supports a distinction between definiteness and determinacy. Previous work has suggested that definite superlative noun phrases like the fewest letters in Gloria received the fewest letters (relative superlative DPs) are semantically indefinite. This paper argues that such DPs are definite (presupposing uniqueness) but not determinate (denoting an individual). We provide new evidence that they are not determinate, and undertake a critical review of the evidence that has previously been used to argue for their indefiniteness, arguing that it is consistent with the hypothesis that they are definite. We argue furthermore that a movement analysis of relative superlatives is not consistent with a treatment of the determiner as definite. We therefore offer an analysis of relative superlative DPs on which they are definite but indeterminate, and the superlative morpheme is interpreted in situ.
\end{abstract}

Keywords: superlatives, relative readings, definiteness, determinacy

\section{Introduction}

In previous work, we have argued for a minimal theory of definiteness, on which the definite article signals a uniqueness presupposition, but no existence presupposition (Coppock \& Beaver 2012, 2014). Our conclusion was based primarily on the behavior of definite descriptions containing exclusive adjectives like only. For example, Liz didn't give the only brilliant talk implies that there is nothing satisfying the description 'only brilliant talk', and this conflicts with the assumption that the definite article encodes an existence presupposition. In this paper, we argue that superlative adjectives, particularly on their so-called relative interpretations, provide additional support for this view.

Relative readings of superlatives are exemplified in the following sentences, taken from the literature (where a subscript $F$ indicates focus-marking).

* We thank Anna Szabolcsi, Dylan Bumford, Cleo Condoravdi, and Christian Josefson for valuable discussion of these issues. This research was supported by Swedish Research Council project 2009-1569 and Riksbankens Jubileumsfond's Pro Futura Scientia program. 
(1) Of her three sisters, [Jean $]_{F}$ bought the most expensive book.

(2) $[\text { Gloria }]_{F}$ read the most books.

(3) There was the least snow on [Tuesday $]_{F}$.

(4) A: How do you win this contest?

B: By putting the tallest plant on the table. (Adapted from Heim 1999)

(5) Who received the fewest letters?

In a sentence like (6), the superlative DP the highest mountain has, in contrast, an absolute reading, meaning 'the mountain that is higher than all other mountains in the United States'.

(6) John climbed the highest mountain in the United States.

The primary issue to be dealt with in this paper is how to interpret the in cases like (1)-(5). Szabolcsi (1986) argued for treating the definite article as vacuous (meaning $a$ ) in such cases, on the basis of facts that we will review below. Heim (1999) and Hackl (2009), proponents of a view on which the superlative morpheme undergoes movement to take scope over the verb on relative readings, are among the authors who have followed Szabolcsi with respect to this assumption. Sharvit \& Stateva (2002) and Krasikova (2012) analyze the superlative morpheme in situ but still do not analyze the definite determiner in the ordinary way. Gawron (1995), Farkas \& É. Kiss (2000), Gutiérrez-Rexach (2006) and Teodorescu (2009) advocate analyses on which -est is interpreted in situ, and do not assume anything unusual for the definite article, but it remains somewhat unclear how to explain the data motivating a treatment of such phrases as indefinite under these analyses; Gawron and Teodorescu do not discuss the issue, and Farkas and Kiss and Gutiérrez-Rexach provide only tentative discussion.

This paper undertakes a critical assessment of the motivations for treating relative superlatives as indefinite at some level, separating two issues: whether relative superlatives are determinate and whether they are (semantically) definite. Following Coppock \& Beaver (2012, 2014), we take definiteness in English to encode a uniqueness presupposition, but assume that definites may be interpreted in three ways: (i) as a predicate (type $\langle e, t\rangle$ ), (ii) determinately (denoting an individual, type $e$ ) or (iii) indeterminately (denoting an existential quantifier, type $\langle\langle e, t\rangle, t\rangle$ ). These distinctions are based on the assumption that the definite article is fundamentally a predicate modifier presupposing uniqueness but not existence. Its meaning can be represented in a three-valued typed lambda calculus as follows:

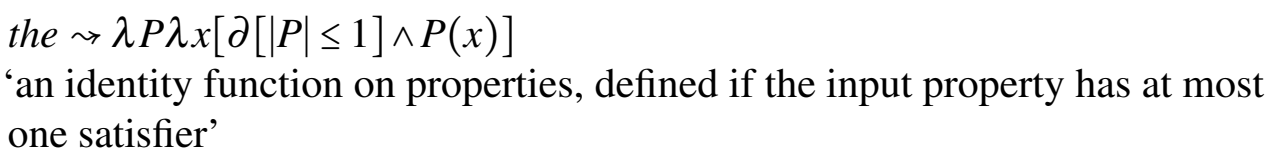


A superlative argument for a minimal theory of definiteness

Here, $\partial$ designates Beaver's (2001) partiality operator; $\partial[|P| \leq 1]$ expresses a presupposition that the number of $P$ s is no greater than 1 . In a predicative sentence like Scott is the author of Waverley, the definite description denotes a predicate which applies directly to the subject. When a definite description is used in argument position, it undergoes one of (at least) two type-shifts, IOTA or EX:

$$
\begin{aligned}
& \operatorname{IOTA}(P) \equiv \iota x[P(x)] \\
& \operatorname{EX}(P) \equiv \lambda Q[\exists x[P(x) \wedge Q(x)]]
\end{aligned}
$$

The IOTA shift produces an expression of type $e$, denoting the unique individual satisfying $P$; the EX shift produces an existential quantifier of type $\langle\langle e, t\rangle, t\rangle$.

This perspective opens up the possibility of a heretofore unconsidered hypothesis regarding relative superlatives: that uniqueness is presupposed, but reference to an individual is not made, so relative superlatives are semantically definite (i.e., carry a uniqueness presupposition), but their denotation is indeterminate rather than determinate. Letting $P$ stand for the descriptive content of a description, the possibilities may be schematized as follows:

i. Indefinite and predicative: $P$

(Type $\langle e, t\rangle$; no presuppositions)

ii. Definite and predicative: $\operatorname{THE}(P) \equiv \lambda x[\partial[|P| \leq 1] \wedge P(x)]$

(Type $\langle e, t\rangle$; presupposes uniqueness but not existence)

iii. Indefinite and indeterminate: $\operatorname{EX}(P) \equiv \lambda Q[\exists x[P(x) \wedge Q(x)]]$

(Type $\langle\langle e, t\rangle, t\rangle$; no presuppositions)

iv. Definite and indeterminate: $\operatorname{Ex}(\operatorname{THE}(P)) \equiv \lambda Q[\exists x[\partial[|P| \leq 1] \wedge P(x) \wedge Q(x)]]$ (Type $\langle\langle e, t\rangle, t\rangle$; presupposes uniqueness but not existence)

v. Definite and determinate: $\operatorname{IOTA}(\operatorname{THE}(P))^{1} \equiv \imath x[P(x)]$

(Type $e$; presupposes uniqueness and existence)

With these finer distinctions at hand, the question may be posed whether the evidence that had been thought to show that relative superlatives are not definite is actually evidence that they are not determinate. As we will show, the empirical evidence for treating relative superlatives as indefinite at some level is not as straightforward as has previously been assumed; much of the evidence is actually consistent with the possibility that definite descriptions are in fact definite and even determinate. We do however provide new evidence, for example from anaphora in opaque contexts,

1 The uniqueness presupposition of $l$ implies the uniqueness presupposition of the; in other words, $\iota x[P(x)]$ is equivalent to $\operatorname{lx}[\partial[|P| \leq 1] \wedge P(x)]$ 
which we take to show that relative superlatives do not denote an individual (i.e., are not determinate). And there are a number of reasons to prefer an analysis on which relative superlatives are definite. Relative superlatives thus appear to constitute a new motivation for distinguishing between definiteness and determinacy.

\section{Motivating the definite-indeterminate analysis}

In this section, we motivate an analysis on which relative superlatives are definite (semantically), but not determinate. An analysis on which relative superlative DPs are semantically definite is clearly preferable prima facie, given that they are morphologically definite. The notion that the definite article can just be treated as an indefinite article would also seem to predict that an indefinite article would also be acceptable with relative superlatives without a change in meaning, and this is not the case:

(10) Gloria climbed the/\#a highest mountain.

The absence of an indefinite article cannot be explained on purely formal morphosyntactic grounds, as superlatives can co-occur with indefinite articles, e.g. the naturally occurring: Every class has a best student, and also a worst student. similar cases are discussed by Herdan \& Sharvit (2006). So if an analysis of the definite article as a marker of semantic definiteness can be maintained, it is clearly to be preferred ceteris paribus.

With a distinction between definiteness and determinacy, it is possible to maintain the assumption that something is definite, without committing to the assumption that it is determinate. Let us consider therefore whether a definite/indeterminate analysis would be preferable to a definite/determinate one. The hypothesis that relative superlative DPs are determinate predicts that they should be associated with a discourse referent that is accessible outside of opaque contexts and hence license anaphora across entailment-cancelling operators. To illustrate, one piece of evidence that Coppock \& Beaver (2014) use to show that definite DPs involving adjectival only can be indeterminate involves whether they license anaphora.

(11) Sue and Jane both scored goals, so Jane didn't score [the only goal $]_{i}$. \#It ${ }_{i}$ wasn't a bicycle-kick, either.

This example shows that the only goal does not pick up or establish a discourse referent that is accessible outside the negation. This supports an analysis of the only goal as indeterminate (an existential quantifier, over which negation takes scope) as opposed to determinate.

Relative superlatives, similarly, do not seem to license anaphora from within opaque (entailment-cancelling) environments. In the following, we use the explicit 
A superlative argument for a minimal theory of definiteness

contrast set out of all of her friends to disambiguate in favor of the relative reading for degree superlatives; definite-marked amount superlatives are already unambiguously relative.

(12) \#Perhaps Gloria climbed the highest mountain out of all of her friends. The prize is a picture of it.

(13) Perhaps Sue climbed the $\{\#$ most, snow-capped $\}$ mountains. I took a picture of them.

These examples do not have a reading where the pronoun refers back to the superlative DP. This implies that the superlative DP does not establish a discourse referent in these cases, contrary to what a determinate analysis would predict.

The following examples provide further evidence that relative superlatives do not pick out a discourse referent that is available outside of opaque contexts. (Note that we guarantee that the degree superlative in (14) can only have a relative reading by making the contrast set explicit with an out of phrase; for (15), the use of an amount superlative suffices to guarantee a relative reading.)

(14) \#You win if out of all the players, you lift the heaviest weight, which is this one.

(15) Sue wanted to see the $\{\#$ most, old $\}$ marble statues, which were the ones I had shown her a picture of.

The unacceptability of non-restrictive modification with relative superlatives can be explained on the grounds that they do not establish a discourse referent, as they would under a determinate analysis.

The following examples show that relative superlatives are consistent with the possibility that there is no satisfier of the description:

(16) Sue wanted to eat the juiciest apple out of all of her friends, but there were no apples.

(17) Sue wanted to eat the \{most, \#large $\}$ apples, but there were no apples.

Under a determinate analysis, existence is presupposed, explaining why the large apples is infelicitous in (17). The fact that denial of existence is compatible with use of relative superlative descriptions in these examples is thus evidence that these superlatives do not have a determinate denotation.

Another prediction of a determinate analysis is that (18) and the superlative variant of (19) should be valid arguments, as the VP-anaphor so should be interpreted with reference to a particular individual picked out by the superlative description. The invalidity of the arguments (as against the validity of the non-superlative variant in (19)) is further evidence for the non-determinacy of relative superlative DPs. 
(18) Sue wanted to see the most famous marble statue out of all of her friends, and so did John. \#Therefore Sue and John wanted to see the same statues.

(19) Sue wanted to see the $\{\#$ most, old $\}$ marble statues, and so did John. Therefore Sue and John wanted to see the same statues.

We conclude that relative superlative DPs are definite but not determinate. In the next section, we will review the evidence that has been adduced in support of the idea that relative superlative DPs are indefinite, and re-evaluate it in light of the distinction between definiteness and determinacy.

\section{Motivations for indefiniteness?}

\subsection{Extraction}

In the classic original paper on this topic, Szabolcsi (1986) gives a range of evidence for the indefiniteness of relative superlatives like the most expensive book in (1). One of the contrasts she points to is the following:

(20) a. ?Who did you take the picture of?

b. Who did you take the best picture of?

The presence of the superlative clearly ameliorates extraction from a definite. The badness of (20a) is thought to be due to a general prohibition against extraction from definite noun phrases (Fiengo \& Higginbotham's (1981) “Specificity Condition”). The acceptability of (20b) is thus taken as evidence that superlative descriptions under relative interpretations are not definite.

It is well-known, however, that extraction from definites is sometimes allowed, quite independently of whether a superlative adjective is present in the noun phrase. Davies \& Dubinsky (2003) discuss a number of such cases. These include some cases involving representational nouns like book and process nouns like coronation:

(21) Who did Ashley write that book about?

(22) Who did Ashley participate in the coronation of?

Even examples that are nearly identical to (20a) can be found in naturally-occurring data. The following is from an edited conversation entitled Has Facebook killed the photo $?^{2}$

2 https://www.academia.edu/2538504/Has_Facebook_Killed_the_Photo_a_conversation_edited_by_ Marvin_Heiferman_ 
A superlative argument for a minimal theory of definiteness

(23) WASOW: I walk down the street, see something, take a picture, send it to my Facebook page and start a dialogue about that, instantly. I did that on my way over here.

SQUIERS: What did you take the picture of?

WASOW: A sign in a window for a sale on shorts.

This suggests that examples like (20a) are not ungrammatical, just hard to find a good context for. Another generalization, shown by the following two examples, is that extraction out of inherently unique definites is typically felicitous.

(24) Who did you take the first/second/next picture of?

(25) Which country is she the Queen of ${ }^{3}$

Since superlative descriptions can reasonably be analyzed as inherently unique, the fact that there is no general ban on extraction from inherently unique definites implies that the extraction data does not bear on the question of whether superlatives are semantically definite. ${ }^{4}$

\subsection{Existential constructions}

Szabolcsi (1986) also points out that relative superlatives can occur as the pivot of an existential sentence, apparently more readily than other definite descriptions.

(26) a. There were the fewest guests yesterday.

b. \#There were the guests yesterday.

Here again, the precise generalization governing the phenomenon is unclear. Certainly there are restrictions on the distribution of definites in existential constructions, but it has been known since Milsark (1977) first observed the so-called definiteness effect in existential constructions that there are exceptions; see for example Ward \& Birner 1995 for discussion. And, indeed, while (26b) may be marked in some contexts, it is clear that it would be acceptable in others, for examples like the following are easy to find:

(27) All the while, there were the guests. Regulars like Don Rickles, Rodney Dangerfield, Bob Newhart.... 5

3 https://www.facebook.com/USGeoChallenge/posts/499937686732717

4 The question of whether superlative descriptions are indeed inherently unique becomes murky for $e x$ situ analyses, because movement of the superlative morpheme leaves behind a description that does not in general satisfy a uniqueness requirement. Furthermore, in such theories the movement of the superlative morpheme would itself be problematic under the twin assumptions that these descriptions are definite and definite descriptions prohibit movement.

5 http://www.today.com/id/6504445/ns/today-today_entertainment/t/ carson-was-everyman-charisma/ 
One might want to relegate cases like (27) to a separate 'use' (for which the labels contextual existentials and list existentials have been used). Then the question becomes what justifies such a distinction, how to characterize this use, and how one can tell what type of use one is dealing with. One test that has been suggested (see McNally 1997: §5.2 and references cited therein) is that list existentials do not form questions well; consider:

(28) A: I'm so overwhelmed! Deadline Monday, mom's birthday tomorrow...

B: \#And were there the guests yesterday?

A question version of the example in (26a), in contrast, is much more natural:

(29) Were there the fewest guests yesterday?

This might be taken as an indication that, if in fact there is such a thing as a 'list existential', (26a) is not one of them. So relative superlatives seem to constitute a class of definites that can occur in existentials on a non-list interpretation.

Relative superlatives are not the only definites that can occur in existentials on a non-list interpretation. Here is a sample of attested examples, along with question versions that we have constructed.

(30) a. It is like the adult time of life, when there is the peak amount of vigor and vitality.

b. Is there the peak amount of vigor and vitality [in the adult time of life $]_{F}$ ?

(31) a. The fetch should generally be determined from the point on the dam where there is the maximum potential for breaching, ...

b. Is there the maximum potential for breaching [at this point $]_{F}$ ?

(32) a. When the two are equal, there is the optimal level of provision for public goods.

b. Is there the optimal level of provision for public goods [when the two are equal $]_{F}$ ?

Interestingly, these all exhibit what might be considered relative readings. For example, the peak amount of vigor and vitality in (30a) can be paraphrased the peak amount of vigor and vitality of any time of life, with a contrast set drawn from the focal alternatives to the adult time of life. So the generalization is not limited to superlatives; it appears that (formally) definite noun phrases under relative interpretations are generally acceptable in non-list existentials. This means that there is a general class of definite descriptions into which relative superlatives fall which can be pivots in non-list existentials. An explanation for this generalization would depend on general theories of both existential constructions and relative readings 
A superlative argument for a minimal theory of definiteness

-we leave these as unsolved puzzles - but we note that the description in each of the above cases is uniquely characterizing, so this evidence appears to be consistent with the idea that the definite article encodes a uniqueness presupposition. ${ }^{6}$ It may even be consistent with an analysis of such noun phrases as determinate, but we currently have no evidence to decide this issue.

\subsection{Relational have}

Another piece of data suggesting that relative superlatives are indefinite are contrasts like the ones in (33), given by Szabolcsi (1986):
a. Who has the smartest sister?
b. ?Who has the sister?
c. Who has a sister?

Example (33a), like (33c), is perfectly acceptable on a relational interpretation of have (where the subject is understood to stand in the 'sister' relation), while (33b) does not seem to be acceptable under a relational interpretation of have.

Here again, relative superlatives pattern like ordinary indefinites and unlike ordinary definites, but the exact generalization governing the phenomenon is not clear, so it is worth asking whether this data really reflects on the definiteness of relative superlatives. As pointed out by Le Bruyn, de Swart \& Zwarts (2013), relational have can co-occur with definite descriptions involving only:

Mary has the only lazy sister.

There are other cases in which a modifier improves a have construction as well:

$$
\text { Mary has }\left\{\begin{array}{c}
\text { the ?(main) responsibility for the project. } \\
\text { the ?(wrong) boyfriend. } \\
\text { the ?(nicer) car. }
\end{array}\right\}
$$

It seems unlikely that the definite article is meaningless in all of these cases. The contrast in (33) therefore does not appear to constitute evidence for the indefiniteness of relative superlatives. ${ }^{7}$

6 Note that these observations are not compatible with Krasikova's (2012) approach to the definiteness of relative superlative DPs, on which uniqueness is evaluated with respect to a property of degrees in DegP, because not all cases of relative readings involve degree constructions.

7 Under several analyses of relational have, it combines with a property; if this analysis is to be adopted, these constructions would involve a predicative interpretation of the DP, rather than a determinate or indeterminate one. 
This concludes our review of the empirical motivations for treating the as $a$ in the literature. ${ }^{8}$ Due to a number of uncertainties, none of the data that we have seen so far clearly allows us to reject the hypothesis that the is definite, and much of the data does not even put the nail in the coffin for a determinate analysis.

\subsection{Incorrect meanings?}

A more indirect argument for the indefiniteness of relative superlatives might run as follows: If they are definite, then we get the wrong interpretation under a movement analysis. And there are independent reasons to favor a movement analysis. Hence they are not definite. In this section, we will show that one premise of this argument is indeed correct: movement analyses do run into trouble when combined with a semantically definite analysis of the definite article, although the issue is subtle. But we question whether there are independent reasons to favor a movement analysis; indeed, there seem to be independent reasons to disprefer one.

Under movement analyses (Heim 1985, 1999; Hackl 2001, 2009), the superlative morpheme undergoes movement so as to scope over the verb. The LF for (36a) is thus as in (36b), where DET is a placeholder for a determiner (either the or $a$ ):

a. Gloria climbed the highest mountain.

b. Gloria [ -est C ] $\lambda d$ climbed DET $d$-high mountain.

Heim (1985: 20) notes regarding her analysis of relative superlatives, "what was the in the sentence had to become $a$ in the LF, or else the meaning would not have seemed quite right." Let us consider what would go wrong with the meaning under this analysis. It turns out not to be obvious that anything would go wrong in basic cases, and exactly what would go wrong depends on the meaning assigned to -est.

We have two versions of -est to consider. Heim (1999) analyzes superlatives in terms of thresholds, in analogous fashion to a Seuren (1973) style analysis of comparatives, while Hackl (2009) uses a comparison among maximum degrees, in a fashion more analogous to von Stechow's (1984) treatment of comparatives. We will gloss these as $-e s t_{\theta}$ and $-e s t_{\max }$ respectively. ${ }^{9}$

8 We have left a couple of observations out of the discussion, including an interesting contrast involving ago: You met Peter \{the fewest years, *those years\} ago. Regarding ago, we observe only that there are other definite descriptions that work in this frame: You met Peter the optimal/perfect number of years ago. Szabolcsi $(2010,2012)$ also notes that relative superlatives license floating each: Who showed the children $\{$ the most, *most of the\} books each? The tentative generalization is that each attaches to "counting quantifiers", but it remains unclear exactly what a counting quantifier is, thus if or how this bears on the definiteness of relative superlatives. Szabolcsi (1986) also gives Hungarian data involving split-NP constructions. The two Hungarian speakers we talked to did not accept split-NP constructions in the first place, so we did not manage to investigate this issue more closely.

9 We assume that these expressions are associated with translations into a typed lambda calculus 
A superlative argument for a minimal theory of definiteness

$-e s t_{\theta} \leadsto \lambda C_{\langle e, t\rangle} \lambda G_{\langle d,\langle e, t\rangle\rangle} \lambda x_{e}$ $\exists d\left[G(x, d) \wedge \forall x^{\prime}\left[\left[C\left(x^{\prime}\right) \wedge x^{\prime} \neq x\right] \rightarrow \neg G\left(x^{\prime}, d\right)\right]\right]$

'there is a degree to which $x$ is $G$ and no distinct $x^{\prime}$ in $C$ is $G$ '

$-e s t_{\max } \leadsto \lambda C_{\langle e, t\rangle} \lambda G_{\langle d,\langle e, t\rangle\rangle} \lambda x_{e}$

$\forall x^{\prime}\left[\left[C\left(x^{\prime}\right) \wedge x^{\prime} \neq x\right] \rightarrow \max d[G(x, d)]>\max d\left[G\left(x^{\prime}, d\right)\right]\right]$

'the maximum degree to which $x$ is $G$ exceeds the maximum degree to which any distinct $x^{\prime}$ in $C$ is $G$,

Regardless of which -est is used, if we have an LF like (36b) where -est has moved to adjoin to VP, $C$ will be a set of alternative mountain climbers, and $G$ will be a relation between individuals and the degrees of height for the mountains they climbed.

The nature of this relation depends on how the determiner is analyzed. Let us compare three treatments of DET: as an existential quantifier $\left(\mathrm{DET}_{\exists}\right)$, as an $t$ operator $\left(\mathrm{DET}_{l}\right)$, and as an identity function on properties presupposing uniqueness, as in (7) above.

$$
\begin{array}{ll}
\text { a. } & \mathrm{DET}_{\exists} \leadsto \lambda P \lambda Q \exists x[P(x) \wedge Q(x)] \\
\text { b. } & \mathrm{DET}_{l} \leadsto \lambda P \operatorname{Pl}[P(x)] \\
\text { c. } & \mathrm{DET}_{\partial[\leq 1]} \leadsto \lambda P \lambda x[\partial[|P| \leq 1] \wedge P(x)]
\end{array}
$$

The last of these can in principle be combined with either an IOTA shift or an EX shift. The former boils down to the $\mathrm{DET}_{l}$ analysis; the EX shift gives us a distinct possibility: definite but indeterminate. These produce the following values for $G$, respectively:

a. indefinite

$\lambda d$ climbed $\mathrm{DET}_{\exists} d$-high mountain $\leadsto$ $\lambda d \lambda x \exists y[\operatorname{MTN}(y) \wedge \operatorname{TALL}(y, d) \wedge \operatorname{CLIMB}(x)(y)]$

b. definite and determinate

$\lambda d$ climbed $\mathrm{DET}_{l} d$-high mountain $\leadsto$ $\lambda d \lambda x[\operatorname{CLIMB}(x)(r y[\operatorname{MTN}(y) \wedge \operatorname{TALL}(y, d)])]$

c. definite and indeterminate

$\lambda d$ climbed $\mathrm{DET}_{\partial[\leq 1]} d$-high mountain $\leadsto$ $\lambda d \lambda x\left[\partial\left(\left|\lambda y^{\prime}\left[\operatorname{MTN}\left(y^{\prime}\right) \wedge \operatorname{TALL}\left(y^{\prime}, d\right)\right]\right| \leq 1\right) \wedge \exists y[\operatorname{MTN}(y) \wedge \operatorname{TALL}(y, d) \wedge\right.$ $\operatorname{CLIMB}(x)(y)]]$

Combining these with -est ${ }_{\theta}$, we get the following predicates to be applied to the subject:

including an operator max with the syntax of $\imath$, forming expressions of type $d$, defined such that $\max d[\phi]$ denotes the greatest degree such that $\phi$. 
a. $\quad-e s t_{\theta} \lambda d$ climbed $\mathrm{DET}_{\exists} d$-high mountain $\leadsto$

$\lambda x \exists d\left[\exists y[\operatorname{MTN}(y) \wedge \operatorname{TALL}(y, d) \wedge \operatorname{CLIMB}(x)(y)] \wedge \forall x^{\prime}\left[\left[C\left(x^{\prime}\right) \wedge x^{\prime} \neq x\right] \rightarrow\right.\right.$ $\left.\left.\neg \exists y\left[\operatorname{MTN}(y) \wedge \operatorname{TALL}(y, d) \wedge \operatorname{CLIMB}\left(x^{\prime}\right)(y)\right]\right]\right]$

b. $\quad$-est ${ }_{\theta} \lambda d$ climbed $\mathrm{DET}_{l} d$-high mountain $\leadsto$ $\lambda x \exists d\left[\operatorname{CLIMB}(x)(\imath y[\operatorname{MTN}(y) \wedge \operatorname{TALL}(y, d)]) \wedge \forall x^{\prime}\left[\left[C\left(x^{\prime}\right) \wedge x^{\prime} \neq x\right] \rightarrow\right.\right.$ $\left.\left.\neg \operatorname{CLIMB}\left(x^{\prime}\right)(\imath y[\operatorname{MTN}(y) \wedge \operatorname{TALL}(y, d)])\right]\right]$

c. $\quad$ est $_{\theta} \lambda d$ climbed $\operatorname{DET}_{\partial[\leq 1]} d$-high mountain $\leadsto$

$\lambda x \exists d\left[\partial\left(\left|\lambda y^{\prime}\left[\operatorname{MTN}\left(y^{\prime}\right) \wedge \operatorname{TALL}\left(y^{\prime}, d\right)\right]\right| \leq 1\right) \wedge \exists y[\operatorname{MTN}(y) \wedge \operatorname{TALL}(y, d) \wedge\right.$ $\operatorname{CLIMB}(x)(y)] \wedge \forall x^{\prime}\left[\left[C\left(x^{\prime}\right) \wedge x^{\prime} \neq x\right] \rightarrow\right.$

$\left.\left.\neg \exists y\left[\operatorname{MTN}(y) \wedge \operatorname{TALL}(y, d) \wedge \operatorname{CLIMB}\left(x^{\prime}\right)(y)\right]\right]\right]$

The formula in (41a) is straightforward. Given the assumption that a mountain of height $d$ is also a mountain of a smaller height $d^{\prime}$ (Heim 1999), (41a) holds of $x$ if $x$ climbed a taller mountain than anyone else in $C$ climbed.

The formula in (41b) has an existentially bound degree variable $d$ inside the $t$ expression $l y[\operatorname{MTN}(y) \wedge \operatorname{TALL}(y, d)])$. The $l$-expression presupposes existence and uniqueness of an entity matching the description. How this presupposition interacts with the existential quantifier over $d$ depends on how presupposition is treated in the logic. Let us assume that in a sentence of the form $\exists x[\phi]$, where $\phi$ contains a presupposition concerning $x$, there need only be one element in the domain that satisfies the presupposition in order for the sentence to have a classical truth value; the presupposition is not imposed on the whole domain (see Muskens 1995, Beaver \& Krahmer 2001, Haug 2013, Coppock \& Beaver 2014). Then, applied to Gloria, what (41b) will presuppose is that there is a degree $d$ such that there is exactly one mountain of height $d$, i.e., a tallest mountain. The sentence asserts that Gloria climbed this mountain and her competitors did not. The definite-indeterminate version (41c) contributes the presupposition that there is a $d$ such that there is at most one mountain of height $d$. If the sentence is true, our protagonist $x$ (e.g., Gloria) climbed a mountain of height $d$. So the truth and definedness conditions of (41c) are the same as those of (41b): that there is a tallest mountain climbed by the protagonist.

The difference in presuppositions between (41a) and (41b)/(41c) has consequences for the analysis of sentences like the following from Heim (1999):

If nobody climbs the highest mountain, the prize is not awarded.

Under (41a), the antecedent is true if the two highest mountains climbed are of equal height. Under (41b), we have a failure to refer in that situation, so the antecedent should be undefined. What complicates the issue is that (42) seems to be roughly on a par with:

If nobody's ever met the king of France, then France might not be a monarchy. 
In other words, to the extent that (42) is acceptable, it could be due to local accommodation of the presupposition under the scope of the negative quantifier. So an analysis presupposing uniqueness does not seem to be unsalvageable for this case. ${ }^{10}$

However, the threshold-based analysis of superlatives would seem run into problems with negative amount superlatives like least and fewest, at least under certain assumptions. Following Hackl (2001), i.a., we assume a silent many of type $\langle d, e t\rangle$, which performs a role in amount superlatives which analogous to the role of the gradable predicate in degree superlatives. Let us suppose that ate fewest popsicles holds of $x$ if there is a degree of popsicle-eating that $x$ did not reach but everyone else did (see Stateva 2000). Then (44a) has the meaning in (44b) under a determinate analysis:

a. Gloria ate the fewest popsicles.

b. determinate analysis:

$$
\begin{aligned}
& \exists d\left[\neg \operatorname { E A T } ( x ) ( \imath y [ { } ^ { * } \operatorname { P O P } ( y ) \wedge \operatorname { M A N Y } ( y , d ) ] ) \wedge \forall x ^ { \prime } \left[\left[C\left(x^{\prime}\right) \wedge x^{\prime} \neq x\right] \rightarrow\right.\right. \\
& \left.\left.\operatorname{EAT}\left(x^{\prime}\right)(\imath y[* \operatorname{POP}(y) \wedge \operatorname{MANY}(y, d)])\right]\right]
\end{aligned}
$$

Again we have an a degree variable in an $\imath$-expression: $\imath y\left[{ }^{*} \operatorname{POP}(y) \wedge \operatorname{MANY}(y, d)\right]$, and again this variable is bound from the outside by an existential quantifier. Suppose Alice ate three popsicles, Bert ate two, and Charlie ate four. In this scenario, the only $d$ for which this expression is defined is 4, assuming downward-monotonicity, i.e., that everyone who ate $n$ popsicles also ate $n-1$ popsicles. Since Alice did not eat 4 popsicles, there is no $d$ such that Alice ate $l$ ( $d$-many popsicles). It is therefore not true that there is a $d$ such that Bert failed to eat $l(d$-many popsicles) and everyone else did. So sentence (44a) incorrectly fails to come out true in this situation. Adopting a definite-but-indeterminate analysis does not help: there is no $d$ such that there is exactly one popsicle plurality numbering $d$ which Alice ate, so again the sentence fails to come out true in this situation. ${ }^{11}$

To summarize the foregoing: It is not clear that a threshold-based, movement analysis of superlatives conflicts with a definite analysis of the determiner in basic

10 Another difference among the meanings is that the presuppositional versions (41b) and (41c) require further contextual restriction on the set of mountains to avoid the conclusion that Gloria climbed the very highest mountain that there is, while (41a) does not. Definite noun phrases need contextual restriction anyway, so this requirement does not seem to have any dangerous empirical consequences.

11 Sharvit \& Stateva (2002: 462) observe that the threshold-based analysis also runs into problems in intensional contexts. Regarding sentences like John needs to climb the highest mountain, they write, "Notice that the main reason for replacing the definite determiner the with the indefinite determiner [...] is semantic. If we leave the definite determiner in [the LF], we get a function from degrees $d$ to sets of individuals who need to climb the unique $d$-high mountain (and consequently, the sentence is predicted to mean that there is a degree $d$ such that John is the only one who needs to climb the unique $d$-high mountain)." This does not produce a valid reading of the sentence. We are leaving intensionality out of the discussion because, as we discuss below, we suspect that the problems here are orthogonal to the analysis of superlatives and definite descriptions. 
cases, but negative amount superlatives do appear to present a challenge for one.

Now let us consider how a max-based, movement analysis of superlatives interacts with definiteness. If we use -est $t_{\max }$ then we get the following analyses, depending on how the determiner is analyzed:

a. $\quad-e s t_{\max } \lambda d$ climbed $\mathrm{DET}_{\exists} d$-high mountain $\leadsto$

$\lambda x \forall x^{\prime}\left[\left[C\left(x^{\prime}\right) \wedge x^{\prime} \neq x\right] \rightarrow\right.$ $\max d[\exists y[\operatorname{MTN}(y) \wedge \operatorname{TALL}(y, d) \wedge \operatorname{CLIMB}(x)(y)]]>$ $\left.\max d\left[\exists y\left[\operatorname{MTN}(y) \wedge \operatorname{TALL}(y, d) \wedge \operatorname{CLIMB}\left(x^{\prime}\right)(y)\right]\right]\right]$

b. $\quad-e s t_{\max } \lambda d$ climbed $\mathrm{DET}_{l} d$-high mountain $\leadsto$ $\lambda x \forall x^{\prime}\left[\left[C\left(x^{\prime}\right) \wedge x^{\prime} \neq x\right] \rightarrow\right.$ $\max d[\operatorname{CLIMB}(x)(l y[\operatorname{MTN}(y) \wedge \operatorname{TALL}(y, d)])]>$ $\left.\max d\left[\operatorname{CLIMB}\left(x^{\prime}\right)(l y[\operatorname{MTN}(y) \wedge \operatorname{TALL}(y, d)])\right]\right]$

c. $\quad-\quad e s t_{\max } \lambda d$ climbed $\operatorname{DET}_{\partial[\leq 1]} d$-high mountain $\leadsto$ $\lambda x \forall x^{\prime}\left[\left[C\left(x^{\prime}\right) \wedge x^{\prime} \neq x\right] \rightarrow\right.$ $\max d\left[\partial\left[\left|\lambda y^{\prime}\left[\operatorname{MTN}\left(y^{\prime}\right) \wedge \operatorname{TALL}\left(y^{\prime}, d\right)\right]\right| \leq 1\right] \wedge \exists y[\operatorname{MTN}(y) \wedge \operatorname{TALL}(y, d) \wedge\right.$ $\operatorname{CLIMB}(x)(y)]>$

$\max d\left[\partial\left[\left|\lambda y^{\prime}\left[\operatorname{MTN}\left(y^{\prime}\right) \wedge \operatorname{TALL}\left(y^{\prime}, d\right)\right]\right| \leq 1\right] \wedge \exists y[\operatorname{MTN}(y) \wedge \operatorname{TALL}(y, d) \wedge\right.$ $\left.\left.\left.\left.\operatorname{CLIMB}\left(x^{\prime}\right)(y)\right]\right]\right]\right]$

As with $-e s t_{\theta}$, the version with an indefinite treatment of the determiner (here, (45a)) is unproblematic. (45b) has an iota expression containing a degree variable that is bound by max. The max expression picks out the highest degree $d$ satisfying the description it contains, which in the first case is the description: $\operatorname{CLIMB}(x)(l y[\operatorname{MTN}(y) \wedge \operatorname{TALL}(y, d)])$. For a given degree $d$, this description will be defined if there is a unique mountain of height $d$, and satisfied if $x$ climbed that mountain. Any $d$ for which there are multiple mountains of height $d$ will make the $\imath$ expression undefined. This is clearly the case if there are two mountains whose maximum height is $d$, and if we again make the assumption that a mountain of height $d$ is also a mountain of a smaller height $d^{\prime}$, then this situation occurs whenever there are multiple mountains at or above height $d$. The only $d$ for which the $l$ expression will be defined is the (maximum) height of the tallest mountain.

How this affects the truth conditions of the sentence depends on how max interacts with partiality. If there are any values for $d$ that make the assumption true, we may assume max selects the highest degree that makes the description true, and disregards all of the degrees yielding false and undefined values. We must also decide what happens if there is no $d$ that makes the description true. In that case it seems reasonable to assume that the max expression is undefined:

$\llbracket \max \delta \phi \rrbracket^{M, g}=$ the degree $d$ that is greatest among those s.t. $\llbracket \phi \rrbracket^{M, g[\delta \rightarrow d]}=1$, if the set of such degrees is non-empty; undefined otherwise 
The problem now is that any $x^{\prime}$ who did not climb the tallest mountain will be such that $\max d\left[\operatorname{CLIMB}\left(x^{\prime}\right)(\imath y[\operatorname{MTN}(y) \wedge \operatorname{TALL}(y, d)])\right]$ will be undefined. We can assume that the undefinedness of this expression makes the whole sentence undefined. If everybody climbed the tallest mountain, then the sentence is false; if anybody failed to climb the tallest mountain, then the sentence is undefined. This means that the body of the universal quantifier $\forall x^{\prime}$ can never have a true value; the sentence is predicted never to be true. So the max-based analysis of -est clashes quite dramatically with the iota analysis of the definite determiner.

The in situ analysis does not have these problems. On an in situ analysis, the definite determiner would combine with '[est $\mathrm{C}] d$-high mountain'. With a determinate analysis, the sentence is predicted to presuppose that there was a highest mountain climbed by Gloria or one of her associates, which would be violated by a tie for highest mountain climbed. The nobody example in (42) is potential counter-evidence to this, but that data is not clear-cut, as discussed above.

A definite-but-indeterminate analysis is even less problematic in combination with an in situ analysis. The determiner as analyzed by Coppock \& Beaver (2012, 2014) contributes the presupposition that there is at most one highest mountain. This is a trivial contribution to the meaning because there is never more than one mountain that is higher than all others. The same holds for negative amount superlatives, by analogy. So with an in situ analysis on which the superlative DP is definite but indeterminate, the truth conditions are just as they would be if it were indefinite.

What we have learned from this exercise is that a movement analysis is not compatible with a treatment of the definite article as definite (uniqueness-presupposing), whether determinate or indeterminate. But an in situ analysis does not produce incorrect meanings when combined with an analysis of the definite article as definite.

Now, given independent reasons to prefer a movement analysis, one might take all of this as indirect evidence for the indefiniteness of relative superlative DPs. One phenomenon that has been shown to be difficult to accommodate under an in situ analysis are Heim's (1999) famous 'upstairs de dicto' readings. Sharvit \& Stateva (2002) do give an analysis of such readings within the context of an in situ analysis, but they use a non-standard analysis of the definite article, so we cannot consider the issue completely resolved. ${ }^{12}$ However, we would like to point out that upstairs de dicto readings are not limited to superlatives. Consider a situation in which little Johnny tells you he is eating a bacon sandwich, and you report:

Johnny thinks he is eating a non-kosher sandwich!

12 Sharvit \& Stateva's (2002) analysis involves an operator IDENT-w* that muddies the worlds so that the bearer of an attitude can't distinguish different properties. This operator also shifts the descriptive content from a property to a set of properties, and the uniqueness presupposition of the is evaluated at the set-of-properties level. 
The de re reading is that there's a particular sandwich Johnny thinks he is eating. The de dicto reading is that Johnny thinks about the sandwich he is eating that it is non-Kosher. Neither of these is correct. What we want is that Johnny thinks about the sandwich he is eating that it has some property, and we know that property entails not being Kosher. In general, we suspect that the 'upstairs de dicto' reading problem is orthogonal to the analysis of superlatives. ${ }^{13}$

Furthermore, there are reasons to disprefer a movement analysis. Farkas \& É. Kiss (2000) observe that when the noun is modified by an overt prepositional phrase as in John visited the largest city in Europe, a relative reading is difficult to get, and this is mysterious under a movement analysis. ${ }^{14}$ Sharvit \& Stateva (2002) point out that movement analyses run into problems with sentences involving negative superlatives, both extensional (e.g., Mary climbed the least high mountain) and intensional (e.g., Mary needs to climb the least high mountain). Teodorescu (2009) gives a number of further arguments against a movement analysis, including the observation that the putative movement of -est would have to disobey island constraints, as shown by the availability of relative readings in examples like John met the man [who had the fastest car] and John met the man [who drove fastest]. So if indeed a movement analysis is not compatible with a treatment of the definite article as definite, the former would seem to be the assumption to discard.

\section{Proposal}

While a number of questions remain open, we conclude that the kind of analysis most strongly supported by the data is one on which relative superlative DPs are definite but indeterminate, and both relative and absolute superlatives are interpreted in situ. The analysis we provide in this section follows these outlines.

Pre-theoretically, we may characterize the difference between relative and absolute readings as follows. Absolute readings involve comparisons solely among entities that lie within the class denoted by the head noun of the superlative phrase; for example, in (6), the highest mountain refers to the mountain that is higher than all other mountains. Relative readings also involve comparisons among a separate set of entities. In (1) for example, Jean is compared to her sisters. Following Gawron (1995), let us use the term contrast set for Jean and her sisters in a case like (1). With relative readings, the contrast set is typically connected to the sentence focus. Call

13 Schwager (2009) considers parallel examples to the 'upstairs de dicto' cases, but her examples do not involve superlatives. This suggests again that such readings constitute a quite general interpretative phenomenon, and tell us little about the analysis of superlatives per se. We note also that her solution does not rely on movement, but on selection of appropriate properties with respect to the world of evaluation and the worlds of the attitude bearer.

14 We are not convinced that a relative reading is impossible here: we find Out of all of his friends, John visited the largest city in Europe to be felicitous, and to have a relative reading. 
the books, snow, plants, letters, and mountains in the above examples the measured entities, as these are the entities whose expensiveness, cardinality, magnitude, height, etc., are measured.

We assume that -est combines with a contrast set $C$ and an association relation $R$ between the contrast set and the set of measured entities. This idea is loosely inspired by Farkas \& É. Kiss's (2000) analysis. ${ }^{15}$

$$
\begin{aligned}
& -e s t \leadsto \lambda C_{\langle e, t\rangle} \lambda R_{\langle e,\langle e, t\rangle\rangle} \lambda G_{\langle d, e t\rangle} \lambda x . \exists y[\partial[R(y, x) \wedge C(y)] \wedge \\
& \left.\exists d\left[G(x, d) \wedge \forall y^{\prime} \forall x^{\prime}\left[\left[R\left(y^{\prime}, x^{\prime}\right) \wedge C\left(y^{\prime}\right) \wedge y \neq y^{\prime}\right] \rightarrow \neg G\left(x^{\prime}, d\right)\right]\right]\right]
\end{aligned}
$$

When the association relation $R$ is trivial (equality), the contrast set is the set of measured entities; this gives us an absolute reading. When the association relation is non-trivial (e.g., ' $x$ climbed $y$ ' or 'there is $y$ on $x$ '), the contrast set is distinct from the set of measured entities, and we have a relative reading. ${ }^{16}$

There are strong restrictions on how $C$ and $R$ may be instantiated. As Farkas \& É. Kiss (2000: 440) note, the association function cannot just be any contextually salient association between individuals: "in a context where it has been established that every individual $c$ in the relevant set $C$ has photographed some set of mountains, [John $n_{F}$ climbed the highest mountain] cannot be interpreted as saying that John climbed a mountain that was higher than any mountain photographed by any other individual in $C$." In order to state the restrictions, let us first establish some terminology. The contrast set is tied to a certain constituent in the sentence: in examples involving focus like (1), this set contains the focus and its alternatives (Jean and her associates), while in (4), the PRO subject of 'putting' is understood to be part of the contrast set, etc. Call the constituent of the sentence that the contrast set is tied to the licensor, following, e.g., Farkas \& É. Kiss (2000). Call the frame of comparison (borrowing Szabolcsi's (1986) term) the result of abstracting over the superlative DP and the licensor, e.g., ' $x$ bought $y$ ', 'there was $y$ on $x$ ', ' $x$ putting $y$ on the table', etc. The association relation $R$ is the relation expressed by the frame of comparison, constrained so that its domain is the contrast set and its domain lies within the class denoted by the head noun (e.g., mountain). We suspect that these constraints may be derivable along the lines suggested by Gawron (1995), who proposes that the

15 An alternative would have been to assume that -est does not combine directly with the association relation, and that this work is done by pragmatics, as it were, as proposed by Teodorescu (2009). On the relative reading of Gloria climbed the highest mountain, the comparison class would be for example 'mountains climbed by members of the contrast set'. Under such an analysis, it is difficult to explain why the sentence would be false if two members of the contrast set both climbed a mountain that was higher than any other mountain climbed by a member of the contrast set; it seems that one needs to make reference to the contrast set.

16 Ideally, the analysis would break the meaning of the superlative down into a comparative part and a superlative part, as in Szabolcsi's (2012) analysis, in light of the results of Bobaljik's (2012) typological study. We leave this for future work. 
parallelism involved in ellipsis resolution also plays a role in the interpretation of relative superlatives.

Given an appropriate contrast set $C$ and $R=$ CLIMBED, the denotation of tallest will be as follows, assuming -est combines directly with tall:

$$
\begin{aligned}
& \lambda x \exists y[\partial[\operatorname{CLIMBED}(y, x) \wedge C(y)] \wedge \\
& \left.\exists d\left[\operatorname{TALL}(x, d) \wedge \forall y^{\prime} \forall x^{\prime}\left[\left[\operatorname{CLIMBED}\left(y^{\prime}, x^{\prime}\right) \wedge C\left(y^{\prime}\right) \wedge y \neq y^{\prime}\right] \rightarrow \neg \operatorname{TALL}\left(x^{\prime}, d\right)\right]\right]\right]
\end{aligned}
$$

This meaning for tallest can combine intersectively with mountain. The definite article combines with tallest mountain, and contributes a presupposition that there is at most one mountain associated with a member of the contrast set that is taller than all others. This is trivially satisfied, so the definite article does not contribute anything to the meaning. Based on the evidence we saw in $\S 2$, relative superlative DPs (typically) undergo an EX shift rather than an IOTA shift, which yields the following meaning for John climbed the tallest mountain, simplifying out the trivial contribution of the definite article:

$$
\begin{aligned}
& \exists x[\operatorname{CLImbed}(\mathrm{J}, x) \wedge \exists y[\partial[\operatorname{CLIMBED}(y, x) \wedge C(y)] \wedge \\
& \left.\exists d\left[\operatorname{TALL}(x, d) \wedge \forall y^{\prime} \forall x^{\prime}\left[\left[\operatorname{CLIMBED}\left(y^{\prime}, x^{\prime}\right) \wedge C\left(y^{\prime}\right) \wedge y \neq y^{\prime}\right] \rightarrow \neg \operatorname{TALL}\left(x^{\prime}, d\right)\right]\right]\right]
\end{aligned}
$$

This correctly captures the meaning of the sentence on a relative interpretation, and is consistent with all of the evidence given above in favor of an analysis on which relative superlative DPs are definite but not determinate.

\section{Summary}

We have argued that relative superlatives are definite, despite putative evidence to the contrary, but indeterminate. They therefore support the distinction between definiteness and determinacy. We have also argued that definite-marked relative superlatives must be interpreted in situ if the definite article contributes a uniqueness presupposition. We offered an analysis along these lines using an analysis of the definite determiner that is entirely general and independently-motivated, and a quite conservative analysis of the superlative, the only innovation being that it relies on both a contrast set and an association relation between the contrast set and the set of measured entities. Why relative superlatives have such an affinity for indeterminate interpretations is a question we must leave for future work.

\section{References}

Beaver, David. 2001. Presupposition and Assertion in Dynamic Semantics. Stanford: CSLI Publications.

Beaver, David \& Emiel Krahmer. 2001. A partial account of presupposition projection. Journal of Logic, Language and Information 10. 147-182. 
A superlative argument for a minimal theory of definiteness

Bobaljik, Jonathan David. 2012. Universals in Comparative Morphology: Suppletion, Superlatives, and the Structure of Words. MIT Press.

Coppock, Elizabeth \& David Beaver. 2012. Weak uniqueness: The only difference between definites and indefinites. In Anca Chereches (ed.), Proceedings of Semantics and Linguistic Theory (SALT) 22, 527-544. Ithaca, NY: CLC Publications.

Coppock, Elizabeth \& David Beaver. 2014. Definiteness and determinacy. Ms., Semantics Archive. http://semanticsarchive.net/Archive/mViOGEzY/.

Davies, William \& Stanley Dubinsky. 2003. On extraction from NPs. Natural Language and Linguistic Theory 21(1). 1-37.

Farkas, Donka \& Katalin É. Kiss. 2000. On the comparative and absolute readings of superlatives. Natural Language and Linguistic Theory 18. 417-455.

Fiengo, Robert \& James Higginbotham. 1981. Opacity in NP. Linguistic Analysis 7(4). 395-421.

Gawron, Jean Mark. 1995. Comparatives, superlatives, and resolution. Linguistics and Philosophy 18. 333-380.

Gutiérrez-Rexach, Javier. 2006. Superlative quantifiers and the dynamics of contextdependence. In Klaus von Heusinger \& Ken Turner (eds.), Where Semantics Meets Pragmatics, 237-266. Oxford: Elsevier.

Hackl, Martin. 2001. Comparative quantifiers. Department of Linguistics Department of Linguistics and Philosophy, Massachusetts Institute of Technology dissertation.

Hackl, Martin. 2009. On the grammar and processing of proportional quantifiers: most vs. more than half. Natural Language Semantics 17. 63-98.

Haug, Dag. 2013. Partial dynamic semantics for anaphora: Compositionality without syntactic coindexation. Journal of Semantics (online first).

Heim, Irene. 1985. Notes on comparatives and related matters. Ms., Semantics Archive. http://semanticsarchive.net/Archive/zc0ZjY0M.

Heim, Irene. 1999. Notes on superlatives. Ms., MIT.

Herdan, Simona \& Yael Sharvit. 2006. Definite and nondefinite superlatives and NPI licensing. Syntax 9(1). 1-31.

Krasikova, Sveta. 2012. Definiteness in superlatives. In Maria Aloni, Vadim Kimmelman, Floris Roelofsen, Galit W. Sassoon, Katrin Schulz \& Matthijs Westera (eds.), Logic, Language and Meaning, vol. 7218/2012, 411-420. Dordrecht: Springer.

Le Bruyn, Bert, Henriëtte de Swart \& Joost Zwarts. 2013. Have, with, and without. In Todd Snider (ed.), Proceedings of Semantics and Linguistic Theory (SALT) 23, 535-548. Ithaca, NY: CLC Publications.

McNally, Louise. 1997. A Semantics for the English Existential Construction. Taylor $\&$ Francis. 
Milsark, Gary. 1977. Toward an explanation of certain peculiarities of the existential construction in English. Linguistic Analysis 3. 1-29.

Muskens, Reinhard. 1995. Meaning and Partiality. Stanford, CA: CSLI Publications. Schwager, Magdalena. 2009. Speaking of qualities. In Ed Cormany, Satoshi Ito \& David Lutz (eds.), Proceedings of Semantics and Linguistic Theory (SALT) 19, 395-412. Ithaca, NY: CLC Publications.

Seuren, Peter. 1973. The comparative. In Ferenc Kiefer \& Nicolas Ruwet (eds.), Generative Grammar in Europe, 528-564. Dordrecht: Reidel.

Sharvit, Yael \& Penka Stateva. 2002. Superlative expressions, context, and focus. Linguistics and Philosophy 25. 453-505.

Stateva, Penka. 2000. In defense of the movement theory of superlatives. In Rebecca Daly \& Anastasia Riehl (eds.), Proceedings of the Eastern States Conference on Linguistics 1999, 215-226. Ithaca, NY: CLC Publications.

Szabolcsi, Anna. 1986. Comparative superlatives. In Naoki Fukui, Tova Rapoport \& Elizabeth Sagey (eds.), Papers in Theoretical Linguistics, 245-265. Cambridge, MA: MITWPL.

Szabolcsi, Anna. 2010. Quantification. Cambridge University Press.

Szabolcsi, Anna. 2012. Compositionality without word boundaries: (the) more and (the) most. In Proceedings of Semantics and Linguistic Theory (SALT) 22, 1-25. Ithaca, NY: CLC Publications.

Teodorescu, Viorica Alexandra. 2009. Modification in the noun phrase: the syntax, semantics, and pragmatics of adjectives and superlatives: University of Texas at Austin dissertation.

von Stechow, Arnim. 1984. Comparing semantic theories of comparison. Journal of Semantics 3. 1-77.

Ward, Gregory \& Betty Birner. 1995. Definiteness and the English existential. Language 71. 722-742.

Elizabeth Coppock

Thunbergsgatan 2

Swedish Collegium for Advanced Study

Uppsala University

75238 Uppsala, Sweden

eecoppock@gmail.com
David Beaver

Center for Liberal Arts, Mailcode B5100

Department of Linguistics

University of Texas at Austin

Austin, TX 78712, USA

dib@mail.utexas.edu 